\title{
Elements of technology of adaptive seed production of vegetable beans in Western Siberia
}

\author{
Yakubenko O.E. ${ }^{1,2 *}$, Parkina O.V. ${ }^{2}$ \\ ${ }^{1}$ Institute of Cytology and Genetics, SB RAS, Novosibirsk, Russia \\ ${ }^{2}$ Novosibirsk State Agrarian University, Novosibirsk, Russia \\ *e-mail: o.e.yakubenko@yandex.ru
}

Today adaptive seed production of crops is a current direction. First of all seed breeders face the problem of adapting varieties to different soil and climatic conditions and reducing the quality of seed production of crops. To introduce vegetable beans into production crops, it is necessary to establish its seed production due to the plasticity of the varieties and the development of elements of the varietal cultivation technology, which will ensure the production of high-quality seed material of the crop. The purpose of the study is to improve the elements of adaptive seed technology for vegetable beans for the conditions of Western Siberia. The object of study is two varieties of common beans Nika and Solnyshko. With the observance of the optimal timing and sowing scheme, it becomes possible to establish seed-growing of vegetable bean in the conditions of the Siberian region. Sowing dates have a significant impact on the formation of the vegetative and generative parts of the plant. With the presence of amicable shoots, the productivity of the crop is significantly increased due to active flowering and bean formation, which creates the conditions for the uniform formation of the crop. Sowing qualities are determined by the term and scheme of sowing culture, taking into account the seed germination energy. When sowing in the third decade of May-the first decade of June, the whole complex of natural factors develops favorably for the growth and development of common bean plants, and it is possible to predict a high seed productivity of the crop. It was established that with an increased seeding rate, a rapid transition to the stage of biological ripeness and, accordingly, increased seed productivity with high quality seed culture is observed. Obtaining a assured yield of high-quality varietal seeds is possible subject to the developed methods of adaptive varietal seed production, recommended for specific growing conditions. 